\title{
INFLUÊNCIA DA COMPOSIÇÃO DOS NANOFIOS PT-IR/C NA ATIVIDADE CATALIÍTICA FRENTE A OXIDAÇÃO ELETROQUÍMICA DE ETANOL EM MEIO ÁCIDO
}

\author{
Gláucia R. O. Almeida ${ }^{\mathrm{a}, \mathrm{b}, \#, \text {, }}$, Eliana M. Sussuchic, Cristiano T. de Meneses ${ }^{\mathrm{d}}$, Luiz P. da Costa ${ }^{\mathrm{e}, \$}$, Giancarlo R. Salazar- \\ Banda $^{\mathrm{a}, \mathrm{b}, \#, *,(1)}$ e Katlin I. B. Eguiluz ${ }^{\mathrm{a}, \mathrm{b}, \#}$ \\ aLaboratório de Eletroquímica e Nanotecnologia, Instituto de Tecnologia e Pesquisa, Universidade Tiradentes, $49032-490$ Aracaju \\ - SE, Brasil \\ bDepartamento de Engenharia de Produção, Universidade Federal de Alagoas, 57200-000 Penedo - AL, Brasil \\ 'Laboratório de Corrosão e Nanotecnologia, Núcleo de Competência em Petróleo e Gás de Sergipe, Universidade Federal de \\ Sergipe, 49100-000 São Cristóvão - SE, Brasil \\ dDepartamento de Física, Universidade Federal de Sergipe, 49506-036 Itabaiana - SE, Brasil \\ eInstituto de Ciências Exatas e Tecnologia, Universidade Federal do Amazonas, 69067-005 Manaus - AM, Brasil
}

Recebido em 08/05/2019; aceito em 15/10/2019 ; publicado em 22/01/2020

\begin{abstract}
ON THE INFLUENCE OF THE COMPOSITION OF Pt-Ir/C NANOWIRES ON THEIR ACTIVITY TOWARDS THE ELECTROCHEMICAL OXIDATION OF ETHANOL IN ACID MEDIA. Carbon-supported platinum-iridium nanowire-shape electrocatalysts containing $20 \%$ of metallic loading were synthesized by chemical reduction method using formic acid as a reducing agent and tested towards the oxidation of ethanol in acidic medium. The developed electrocatalysts were synthesized with the following compositions: $\mathrm{Pt}_{0.9} \mathrm{Ir}_{0.1} / \mathrm{C}, \mathrm{Pt}_{0.8} \mathrm{Ir}_{0.2} / \mathrm{C}, \mathrm{Pt}_{0.7} \mathrm{Ir}_{0.3} / \mathrm{C}, \mathrm{Pt}_{0.6} \mathrm{Ir}_{0.4} / \mathrm{C}$ and $\mathrm{Pt}_{0.5} \mathrm{Ir}_{0.5} / \mathrm{C}$ using for comparison the commercial $\mathrm{Pt} / \mathrm{C} \mathrm{Alfa}$ Aesar catalyst. Physico-chemical characterization was performed using X-ray diffraction (XRD), transmission electron microscopy (TEM), and X-ray fluorescence (XRF, for chemical composition) techniques. All the nanowires present a face-centered cubic crystalline structure of the platinum with modifications in the lattice parameter due to the inclusion of iridium. The XRF analyzes show that the theoretical compositions of the synthesized catalysts are very close to the experimental nominal values. TEM analyses showed the formation of nanowires by the synthesis method used. The electrochemical results on the oxidation of ethanol show that the addition of a second metal to the platinum in the composition of the nanowires increases the catalytic activity of the electrode. The nanowire catalysts with low Ir content $\left(\mathrm{Pt}_{0.9} \mathrm{Ir}_{0.1} / \mathrm{C}, \mathrm{Pt}_{0.8} \mathrm{Ir}_{0.2} / \mathrm{C}, \mathrm{Pt}_{0.7} \mathrm{Ir}_{0.3} / \mathrm{C}\right)$ present the best outcomes under the analyzed conditions and are promising for application as anodes in direct ethanol fuel cells.
\end{abstract}

Keywords: nanowires; ethanol electrooxidation; fuel cells; electrocatalysts

\section{INTRODUÇÃO}

O desenvolvimento sustentável tornou-se um dos assuntos mais discutidos atualmente devido ao modelo capitalista adotado e ao consumismo desenfreado da sociedade. Essa explosão no consumo, juntamente com o crescimento demográfico faz com que a demanda pelos recursos naturais cresça de forma exponencial prejudicando assim a qualidade de vida das pessoas. Os combustíveis fósseis são ainda a principal fonte de energia utilizada nos mais diversos tipos de máquinas, atividades industriais e motores a combustão interna. ${ }^{1}$ Esses combustíveis são um dos principais degradadores ambientais associados com a emissão de gases de efeito estufa, principalmente o $\mathrm{CO}_{2}$, produzido durante a queima para a geração de energia, pois correspondem cerca de $80 \%$ do consumo global, sendo $58 \%$ desse total consumido pelo setor de transportes. ${ }^{2}$ Do período da revolução industrial até os dias atuais os níveis de $\mathrm{CO}_{2}$ aumentaram de 280 ppm para uma concentração diária de $400 \mathrm{ppm}^{3}$

As células a combustível surgem como uma fonte alternativa para a substituição de fontes de energia baseadas nos combustíveis fósseis. Sua operação depende da utilização de um combustível em que se converte a energia química em energia elétrica. Várias células a combustível são propostas incluindo as células a combustível

*e-mail: gianrsb@gmail.com

"Curso de Engenharia de Produção

"Programa de Pós-graduação em Engenharia de Processos

§rograma de Pós-graduação em Química de metanol direto (DMFCs), ${ }^{4}$ etanol direto (DEFCs), ${ }^{5}$ óxido sólido (SOFCs), ${ }^{6}$ alcalinas, ${ }^{7}$ etc. Elas apresentam diversas vantagens, dentre elas baixas emissões de poluentes durante a geração de eletricidade e proporcionam maior segurança energética. ${ }^{8,9} \mathrm{O}$ avanço na tecnologia das células a combustível é devido aos diversos estudos na área de eletrocatálise. ${ }^{10} \mathrm{O}$ hidrogênio é um combustível que tem sido bastante utilizado nesse tipo de tecnologia devido à sua flexibilidade de obtenção, mas seu uso se torna limitado devido a problemas relacionados com os sistemas operacionais e de infraestrutura por ser um gás altamente explosivo e de difícil manipulação. ${ }^{11}$

Diante dessa problemática cresce o interesse em utilizar álcoois diretamente como combustíveis, sendo que o uso de etanol tem recebido grande atenção nos últimos anos por ser um combustível renovável, com características de baixa emissão de poluente, facilidade de obtenção (com possibilidade de produção em grande quantidade através da biomassa). ${ }^{12} \mathrm{O}$ metanol por sua vez tem sido amplamente empregado, pois apresenta alta solubilidade em eletrólitos aquosos, facilidade de transporte e fornecimento para o mercado consumidor sem precisar de mudança na infraestrutura, como no caso do hidrogênio. ${ }^{4}$

Em processos de eletro-oxidação de álcoois, o aumento do número de átomos de carbono resulta em reações paralelas que podem levar à formação de $\mathrm{CO}_{2}$ e de vários intermediários que são formados e adsorvidos na superfície do catalisador diminuindo assim a cinética de oxidação do álcool. ${ }^{5,13}$

O maior desafio no desenvolvimento das células a combustível de etanol é desenvolver catalisadores que melhorem a cinética de reação e minimizem a formação de intermediários. A platina é o 
principal catalisador utilizado nesse tipo de células a combustível, mas é altamente contaminada durante a oxidação do álcool.

Em catalisadores contendo Pt-Ir, durante a adsorção dissociativa de etanol nos sítios ativos da Pt, o Ir promove a adsorção de espécies oxigenadas facilitando a oxidação de $\mathrm{CO} .{ }^{14} \mathrm{Cao}$ et al.${ }^{15}$ estudaram a eletrooxidação de etanol utilizando $\mathrm{Ir}$ e $\mathrm{Sn}$ e mostraram que $\mathrm{Ir}_{3} \mathrm{Sn} / \mathrm{C}$ obteve uma taxa mais elevada de oxidação de etanol em comparação ao catalisador $\mathrm{Pt}_{3} \mathrm{Sn} / \mathrm{C}$ em baixos potenciais a uma temperatura de $90{ }^{\circ} \mathrm{C}$, isso pode ser explicado devido à presença de Sn e Ir promoverem a eletro-oxidação de espécies adsorvidas na superfície do catalisador a $\mathrm{CO}$.

Tayal et al. ${ }^{16}$ estudaram a combinação de nanocatalisadores bimetálicos e trimetálicos de $\mathrm{Pt}$, Ir, Sn preparados pelo método de redução por impregnação em carbono Vulcan XC-72, para melhorar a eletrooxidação de etanol. Os nanocatalisadores Pt-Ir-Sn/C exibiram o maior desempenho entre todos os catalisadores preparados com densidade de potência de $29 \mathrm{~mW} \mathrm{~cm}{ }^{-2}$ em células a combustível de etanol direta operando a $90{ }^{\circ} \mathrm{C}$.

Os eletrocatalisadores de platina com morfologia 0D (nanopartículas) geralmente apresentam um grande número de átomos de baixa coordenação, ocasionando assim um grande número de defeitos na sua superfície. Entretanto, pesquisadores tem mostrado que nanoestruturas de platina com morfologia 1D (nanofios) mantêm segmentos cristalinos individuais alongados com planos cristalinos que são por sua vez conectados pelos contornos dos grãos formando uma estrutura de nanofios. A forma estrutural e as propriedades eletrônicas dos eletrocatalisadores 1D de platina podem estar diretamente ligadas a um aumento considerável no desempenho catalítico da reação de redução do oxigênio, assim como também da reação de oxidação de etanol. ${ }^{17}$ Estas características podem melhorar a sua utilização em diferentes aplicações, tais como eletrocatalisadores para sistemas de células a combustível para conversão de energia. ${ }^{18-21}$

Recentemente, nanocatalisadores de Pt-Ir de diferentes morfologias, incluindo nanofios foram desenvolvidos e testados na reação de desprendimento de oxigênio. Os nanocatalisadores foram mais eficientes do que o catalisador $\mathrm{Ir} / \mathrm{C}$ comercial e a atividade catalítica diminui com a proporção de espécies $\mathrm{IrO}_{x}$ no mesmo. ${ }^{22}$

Neste trabalho, foram sintetizados nanofios de Pt-Ir suportados em carbono em diferentes proporções pelo método de redução química sem adição de surfactantes, para estudar a reação de eletro-oxidação de etanol em meio ácido. Os nanocatalisadores foram caracterizados por voltametria cíclica, difração de raios X (DRX), microscopia eletrônica de transmissão (MET) e fluorescência de raios X (FRX).

\section{PROCEDIMENTO EXPERIMENTAL}

O substrato utilizado na síntese dos nanofios de Pt-Ir foi o carbono (Vulcan $\left.{ }^{\circledR} \mathrm{XC} 72\right)$. O ácido hexacloroplatínico $\left(\mathrm{H}_{2} \mathrm{PtCl}_{6} \cdot 6 \mathrm{H}_{2} \mathrm{O}\right.$ - Sigma-Aldrich ${ }^{\circledast}, 37,5 \%$ de Pt) e cloreto de irídio hidratado $\left(\mathrm{IrCl}_{3}\right.$. $\mathrm{xH}_{2} \mathrm{O}$ - Sigma-Aldrich ${ }^{\circledR}$ ) foram utilizados como precursores metálicos. Os nanofios (20\% de carga metálica) e diferentes composições entre Pt e Ir foram sintetizados de acordo com o método de redução química. ${ }^{19} \mathrm{O}$ ácido fórmico (Vetec, 98-100\% de pureza) foi utilizado como agente redutor. Todos os experimentos foram realizados a temperatura ambiente $\left(25^{\circ} \mathrm{C}\right)$ em solução aquosa e na ausência de surfactantes.

Para o crescimento dos catalisadores Pt-Ir no substrato de carbono, $\mathrm{o} \mathrm{IrCl}_{3} \cdot \mathrm{xH}_{2} \mathrm{O}$ foi dissolvido em água ultrapura obtida por um sistema Gehaka modelo MS 2000, para posterior adição do $\mathrm{H}_{2} \mathrm{PtCl}_{6} \cdot 6 \mathrm{H}_{2} \mathrm{O}$. Em seguida, uma quantidade de carbono (Vulcan ${ }^{\circledR}$ XC72) foi adicionado sob agitação de $100 \mathrm{rpm}$ por $30 \mathrm{~min}$ e por último o ácido fórmico ( $\mathrm{HCOOH}$, Vetec, $98-100 \%$ de pureza) foi inserido como agente redutor dos íons metálicos sobre o substrato de carbono. A solução ficou armazenada por $72 \mathrm{~h}$ a temperatura ambiente. Após esse tempo o sólido resultante foi filtrado e lavado várias vezes com água ultrapura com o auxílio de uma bomba a vácuo modelo PRISMATEC 131, utilizando membrana em ésteres de celulose (com poros de $47 \mu \mathrm{m}$ ) da Millipore. Posteriormente levado à estufa para secagem a $60^{\circ} \mathrm{C}$ por $30 \mathrm{~min}$.

As análises de MET foram realizadas utilizando um microscópio eletrônico JEOL 2100 HTP JEM em 200 kV. As medidas de DRX foram realizadas utilizando um difratômetro (PANalytical EMPYREAN) com geometria Bragg-Bretano, com detector PIXcel ${ }^{\text {ld }}$, operando com radiação $\mathrm{Cu} \mathrm{K} \alpha(\lambda=0.15406 \mathrm{~nm})$ e com aplicação de tensão e corrente, respectivamente, de $40 \mathrm{kV}$ e $40 \mathrm{~mA}$. Os parâmetros utilizados (varredura angular $2 \theta=20^{\circ}-90^{\circ}$, com passo de $0,5^{\circ} \mathrm{min}^{-1}$ foram mantidos constantes durante todas as análises. Todos os padrões de difração foram analisados utilizando o programa Philips X'pertHighScore Plus e o banco de dados cristalográfico JCPDS. O refinamento Rietveld foi realizado em todas medidas de difração de raios $\mathrm{X}$, considerando apenas a fração cristalizada, a fase da nanoliga. Para isso foi utilizado o programa DBWS 9807 conforme implementado na interface DBWSTools2.3. ${ }^{23}$

As análises eletroquímicas foram realizadas em um potenciostato/ galvanostato modelo Autolab PGSTAT302N acoplado a um computador. Os perfis voltamétricos dos diferentes eletrocatalisadores foram obtidos em uma célula eletroquímica de um compartimento com o corpo confeccionado em vidro Pirex ${ }^{\circledR}$, contendo um eletrodo de referência de hidrogênio preparado na mesma solução (EHMS), um contra eletrodo de platina de $1 \mathrm{~cm}^{2}$ e o eletrodo de carbono vítreo. Os experimentos foram realizados em solução ácida de $\mathrm{H}_{2} \mathrm{SO}_{4} 0,5 \mathrm{~mol} \mathrm{~L}-1$ saturada com nitrogênio $\left(\mathrm{N}_{2}\right)$. O eletrodo de trabalho para cada teste foi preparado a partir de uma camada ultrafina de suspensão sobre um eletrodo de carbono vítreo. Para isso, foi utilizada uma suspensão contendo $0,0032 \mathrm{~g}$ do catalisador, $1000 \mu \mathrm{L}$ de álcool isopropílico (Sigma-Aldrich ${ }^{\circledR}$ ) e $30 \mu \mathrm{L}$ de náfion em solução (Aldrich Chemical, solução a 0,05 wt\% em álcoois alifáticos).

A mistura resultante foi levada ao banho de ultrassom (Ultracleaner $1450 \mathrm{~A}$ ) até se tornar homogênea e, em seguida, uma camada de $5 \mu \mathrm{L}$ foi depositada no eletrodo de carbono vítreo. Em seguida seco ao ar ambiente e hidratado com água ultrapura durante $1 \mathrm{~min}$, obtendo-se assim um filme fino de catalisador. Todos os potenciais dos eletrodos de trabalho obtidos foram referenciados ao EHMS.

Os testes de atividades catalíticas para a eletrooxidação de etanol foram realizados em meio ácido $\left(\mathrm{H}_{2} \mathrm{SO}_{4}\right) 0,5 \mathrm{~mol} \mathrm{~L}^{-1}$ saturado com nitrogênio $\left(\mathrm{N}_{2}\right)$ e a uma velocidade de varredura de $20 \mathrm{mV} \mathrm{s}^{-1}$. As análises da eletrooxidação de etanol foram comparadas com a $\mathrm{Pt} / \mathrm{C}$ da Alfa Aesar. Todas as análises foram realizadas em triplicata e com um volume da célula eletroquímica de $64 \mathrm{~mL}$.

Para o cálculo da área eletroativa, utilizou-se a área abaixo do pico de oxidação de $\mathrm{CO}$, pois a oxidação de uma monocamada de CO linearmente adsorvida na $\mathrm{Pt}^{24}$ envolve uma carga de $420 \mu \mathrm{C} \mathrm{cm}^{-2}$ e a oxidação de uma monocamada de $\mathrm{CO}$ em $\operatorname{Ir}^{25}$ gera uma densidade de carga de $287,7 \mu \mathrm{C} \mathrm{cm}^{-2}$. Todos os resultados eletroquímicos reportados neste trabalho foram normalizados pela área eletroativa calculada a partir da voltametria de desorção de $\mathrm{CO}$ e referenciados usando o EHMS.

\section{RESULTADOS E DISCUSSÃO}

\section{Caracterização físico-química}

Os padrões de difração de raios X experimentais e calculados através do refinamento Rietveld dos eletrocatalisadores de Pt-Ir/C são mostrados na Figura 1. A identificação das fases foi realizada comparando os picos obtidos com os padrões presentes no banco de 
dados do Joint Committe on Powder Diffraction Standards (JCPDS). O pico de difração alargado que aparece próximo a $2 \theta=25^{\circ}$ corresponde a contribuição da fase do carbono Vulcan ${ }^{\circledR} \mathrm{XC72R}$, composto suporte das nanoligas, sendo este observado em todos os padrões de raios X. Os picos de difração em aproximadamente $40^{\circ}, 46^{\circ}, 67^{\circ} \mathrm{e}$ $80^{\circ}$ são referentes à família de planos cristalinos $\{111\},\{200\},\{220\}$ e $\{311\}$ correspondentes à estrutura cúbica da platina com grupo espacial Fm-3m (identificada pela ficha JCPDS - 01-087-0636). Como a estrutura cristalina do irídio é isomorfa e seus valores de parâmetros de rede são muito próximos ao da estrutura da platina, os picos de difração de ambas as fases estão convolucionados. As análises de difração de raios X mostram que, na presença de Ir, os padrões da Pt não sofreram significativas modificações em seus picos com o aumento da razão Ir/Pt. No entanto, uma análise mais precisa dos refinamentos aponta um leve aumento nos parâmetros de rede com o aumento da concentração de Ir, e este comportamento pode estar associado ao fato do raio atômico do Ir ser suavemente maior do que o da Pt. Esses valores estão apresentados na Tabela 1, os quais são condizentes com os relatados por Tayal et al. ${ }^{16}$



Figura 1. Padrões de difração de raios X experimentais (pontos) e calculados (linha vermelha) e suas diferenças (linha verde) para os eletrocatalisadores preparados pelo método de redução química

Para estimar o tamanho médio dos cristalitos a largura à meia altura da reflexão correspondente do pico (220) foi utilizada, pois minimiza qualquer efeito da contribuição amorfa do carbono. ${ }^{26}$ Para isso a equação de Scherrer foi aplicada e os resultados estão apresentados na Tabela 1. Sendo que os tamanhos dos cristalitos variaram de 4,1 a 4,6 nm, não apresentando significantes mudanças em seus valores. Os parâmetros de rede cristalina foram 0,3920, 0,3936, 0,3930, 0,3946 e 0,3951 nm nos nanofios de $\mathrm{Pt}_{0,9} \mathrm{Ir}_{0,1} / \mathrm{C} ; \mathrm{Pt}_{0,8} \mathrm{Ir}_{0,2} / \mathrm{C}$; $\mathrm{Pt}_{0,7} \mathrm{Ir}_{0,3} / \mathrm{C} ; \mathrm{Pt}_{0,6} \mathrm{Ir}_{0,4} / \mathrm{C} ; \mathrm{Pt}_{0,5} \mathrm{Ir}_{0,5} / \mathrm{C}$, respectivamente.

Tabela 1. Parâmetros estruturais obtidos pelas técnicas de DRX e FRX para os eletrocatalisadores sintetizados

\begin{tabular}{cccc}
\hline Eletrocatalisadores & $\begin{array}{c}\text { Tamanho do } \\
\text { cristalito (nm) }\end{array}$ & $\begin{array}{c}\text { Parâmetro } \\
\text { de rede (nm) }\end{array}$ & $\begin{array}{c}\text { Pt-Ir } \\
\text { Experimental (\%) } \\
\text { (FRX) }\end{array}$ \\
\hline $\mathrm{Pt}_{0,9} \mathrm{Ir}_{0,1} / \mathrm{C}$ & 4,6 & $0,3920(8)$ & $91,5: 8,5$ \\
$\mathrm{Pt}_{0,8} \mathrm{Ir}_{0,2} / \mathrm{C}$ & 4,0 & $0,3936(1)$ & $82,3: 17,7$ \\
$\mathrm{Pt}_{0,7} \mathrm{Ir}_{0,3} / \mathrm{C}$ & 4,1 & $0,3930(1)$ & $72,6: 27,4$ \\
$\mathrm{Pt}_{0,6} \mathrm{Ir}_{0,4} / \mathrm{C}$ & 4,0 & $0,3946(1)$ & $61,4: 38,6$ \\
$\mathrm{Pt}_{0,5} \mathrm{Ir}_{0,5} / \mathrm{C}$ & 3,5 & $0,3951(1)$ & $53,4: 46,6$ \\
\hline
\end{tabular}

As imagens de microscopia eletrônica de transmissão indicam a formação dos nanofios uniformemente dispersos no suporte de carbono para todas as composições sintetizadas (Figura 2).

A composição química dos nanofios preparados foi determinada por FRX a fim de definir quantitativamente as proporções atômicas médias dos elementos presentes na amostra, e verificar se as proporções sintetizadas estão conforme aquelas desejadas. Pode-se observar na Tabela 1 que os valores médios encontrados experimentalmente são próximos aos das composições nominais.

Os nanofios apresentam diâmetro médio de 4-5 nm e comprimento da ordem de 10-20 nm, Pode-se também observar a presença de nanopartículas nos catalisadores com maior proporção de Ir, indicando que este elemento não forma nanofios tão facilmente quanto a Pt, como já demonstrado anteriormente para nanofios de $\mathrm{PtRu} / \mathrm{C} .^{19,21}$

\section{Caracterização eletroquímica}

Voltamogramas cíclicos dos nanocatalisadores em solução de $0,5 \mathrm{~mol} \mathrm{~L}^{-1} \mathrm{de}_{2} \mathrm{SO}_{4}$ foram obtidos para determinar o perfil voltamétrico dos nanocatalisadores. A Figura 3 mostra as voltametrias cíclicas obtidas dos catalisadores contendo nanofios de Pt-Ir em solução de $\mathrm{H}_{2} \mathrm{SO}_{4} 0,5 \mathrm{~mol} \mathrm{~L}^{-1}$ saturada em nitrogênio a $20 \mathrm{mVs}^{-1}$ a temperatura ambiente e usando uma janela de potencial de 0,50 a 0,80 V vs. EHMS. O perfil obtido se assemelha ao perfil da platina policristalina. ${ }^{27}$

Foram observados picos redox típicos, associados à adsorção/ dessorção de $\mathrm{H}_{2}$ que é formado pela oxidação do hidrogênio absorvido (varredura anódica) e redução de íons de $\mathrm{H}^{+}$presentes na solução (varredura catódica) entre 0,05-0,3 V vs. EHMS. O eletrodo entre 0,3 e $0,7 \mathrm{~V}$ se comporta como idealmente polarizado apresentando apenas correntes capacitivas correspondentes à acomodação dos íons na dupla camada. Os nanofios $\mathrm{Pt}_{0,5}-\mathrm{Ir}_{0,5} / \mathrm{C}$ mostraram picos de adsorção/dessorção de hidrogênio bastante reduzidos, fato que pode afetar significativamente a atividade eletrocatalítica deste catalisador. Neste sentido, as medidas de DRX mostraram que este catalisador apresentou picos de difração com intensidades menores quando comparados com os outros nanofios. Este comportamento também pode ser atribuído ao recobrimento dos sítios ativos da platina pelo irídio, pois a platina pode ser reduzida numa velocidade maior que o irídio.

\section{Desorção oxidativa de CO}

A oxidação de $\mathrm{CO}$ a platina obedece ao modelo cinético de Langmuir-Hinshelwood, em que o mesmo sítio ativo é disputado pelo $\mathrm{O}_{2}$ e $\mathrm{CO}$, diminuindo a taxa de reação quando a quantidade de $\mathrm{CO}$ é aumentada. Quando um segundo metal é adicionado à platina geram dois sítios ativos fazendo com que seja minimizado esse efeito competitivo, pois o $\mathrm{CO}$ é oxidado no sítio da platina e o $\mathrm{O}_{2}$ é adsorvido de forma dissociada no sítio do segundo metal. ${ }^{28}$

A Figura 4 apresenta a remoção de $\mathrm{CO}$ das nanopartículas do catalisador comercial (Pt/C, Alfa Aesar). O início da oxidação do CO, bem como o potencial de pico de remoção $(\approx 0,8 \mathrm{~V})$ estão de acordo com estudos prévios observados na literatura em meio de $\mathrm{H}_{2} \mathrm{SO}_{4}{ }^{29}$ Para os nanofios $\mathrm{Pt}_{0,8} \mathrm{Ir}_{0,2} / \mathrm{C}, \mathrm{Pt}_{0,7} \mathrm{Ir}_{0,3} / \mathrm{C} \mathrm{e} \mathrm{Pt}_{0,6} \mathrm{Ir}_{0,4} / \mathrm{C}$ o início de oxidação de $\mathrm{CO}$ teve um deslocamento de aproximadamente $100 \mathrm{mV}$ para valores mais negativos quando comparados à $\mathrm{Pt} / \mathrm{C}$ comercial. O pico máximo nestes catalisadores é também deslocado em $10 \mathrm{mV}$ para valores mais negativos do que a $\mathrm{Pt} / \mathrm{C}$. O catalisador $\mathrm{Pt}_{0.5} \mathrm{Ir}_{0.5} / \mathrm{C}$ apresenta pico de oxidação de $\mathrm{CO}$ em aproximadamente $0,8 \mathrm{~V}$, valor semelhante ao apresentado pelo catalisador comercial. Este comportamento pode se dever à quantidade elevada de Ir presente no catalisador, diminuindo assim a quantidade de sítios ativos da platina.

A oxidação de CO na Pt/C comercial apresenta um pré-pico e um pico de oxidação principal, fato que não é verificado nos nanofios de 




(a)



(d)



(b)



(e)



(c)

Figura 2. Micrografias eletrônicas de transmissão dos nanofios sintetizados: $P t_{0,9} I r_{0,1} / C(a) ; P t_{0,8} I r_{0,2} / C(b) ; P t_{0,7} I r_{0,3} / C(c) ; P t_{0,6} I r_{0,4} / C(d) ;$ e $P t_{0,5} I r_{0,5} / C(e)$.



Figura 3. Voltamogramas cíclicos (segundo ciclo) realizados sobre os nanofios $P t_{0,9} I r_{0,1} / C ; P t_{0,8} I r_{0,2} / C ; P t_{0, I} I r_{0,3} / C ; P t_{0,6} I r_{0,4} / C ; P t_{0,5} I r_{0,5} / C$ no eletrólito de suporte $\left.\left(\mathrm{H}_{2} \mathrm{SO}_{4} 0,5 \mathrm{~mol} \mathrm{~L}\right)^{l}\right)$ a v $=20 \mathrm{mV} \mathrm{s}^{l}$.

Pt-Ir/C. Quando o CO é adsorvido na região de potenciais de adsorção/dessorção de hidrogênio, um pré-pico de oxidação acontece em aproximadamente $0,5 \mathrm{~V} v s$. EHMS sugerindo uma cobertura parcial da superfície do eletrodo com o $\mathrm{CO}$ adsorvido devido a traços de $\mathrm{CO}$ na solução. $\mathrm{O}$ segundo pico acontece em aproximadamente $0,8 \mathrm{~V}$ e corresponde ao restante da carga de oxidação.

A ausência de uma resposta abaixo de $0,5 \mathrm{~V}$ sugere uma cobertura completa da superfície do eletrodo com o CO adsorvido, apresentando apenas um pico de oxidação como comprovado por Ochal et al., ${ }^{30}$ Já os nanofios de Pt-Ir apresentaram pico de oxidação entre $0,78-0,80 \mathrm{~V}$ quando isso acontece pode ser explicado que o CO é adsorvido na região de potenciais de dupla camada elétrica, onde ocorre os processos predominantemente capacitivos e depois é eliminado da solução inexistindo a presença do pré-pico na região de adsorção/dessorção de hidrogênio. ${ }^{31}$

A presença de picos superpostos observados em alguns catalisadores binários pode estar relacionada à oxidação de $\mathrm{CO}$ em diferentes planos cristalinos provavelmente presentes em nanofios. O primeiro pico pode ser atribuído à oxidação de CO no plano (111), enquanto o segundo pico se refere à oxidação sobre o plano (100). ${ }^{29}$

Em estudos realizados por $\mathrm{Qu}$ et al., ${ }^{32}$ eletrocatalisadores contendo Pt e Ir com diferentes rações molares para $\mathrm{Pt}$ e $\mathrm{Ir}(\mathrm{Pt} / \mathrm{C}$, $\mathrm{Pt}_{3} \mathrm{Ir}_{1}, \mathrm{Pt}_{1} \mathrm{Ir}_{3} / \mathrm{C}$ e Ir/C) foram sintetizados pelo método de poliol modificado. Foi observado que o início do pico de oxidação do $\mathrm{CO}$ para a Pt/C e Ir/C foi em torno de 0,6 V. Embora o Ir apresente caráter oxófilico, ele não consegue obter uma cinética mais rápida em comparação à Pt/C para o potencial de dessorção de CO. Já os eletrocatalisadores $\mathrm{Pt}_{3} \mathrm{Ir}_{1} / \mathrm{C}$ e $\mathrm{Pt}_{1} \mathrm{Ir}_{3} / \mathrm{C}$ exibiram um menor potencial de início de oxidação a 0,25 e $0,30 \mathrm{~V}$, respectivamente. A cinética de remoção do $\mathrm{CO}$ em eletrocatalisadores à base de $\mathrm{Pt}$ pode ser determinada pela cobertura de dois intermediários adsorvidos durante a reação, o $\mathrm{CO}_{\mathrm{ads}}$ e o $\mathrm{OH}_{\mathrm{ads}} \cdot{ }^{33}$ Uma explicação para a cinética mais rápida de remoção de $\mathrm{CO}$ em ligas bimetálicas de Pt-Ir pode ser deduzida através do efeito ligante do Ir à estrutura eletrônica da Pt, onde a inclusão de átomos de Ir na Pt pode levar a um deslocamento negativo no centro da banda $5 \mathrm{~d}$ da platina causado pelas interações na rede entre os átomos de $\mathrm{Pt}-\mathrm{Ir}^{34}$ resultando numa energia de adsorção mais fraca para ligação Pt e CO, levando assim à aceleração na cinética das ligas Pt-Ir.

De acordo com Shivhare et al., ${ }^{35}$ em baixos potenciais grupos 



Figura 4. Voltamogramas cíclicos para oxidação de CO sobre os eletrodos: Pt/C Alfa Aesar; nanofios $P t_{0,9} I r_{0, I} / C ; P t_{0,8} I r_{0,2} / C ; P t_{0,7} I r_{0,3} / C ; P t_{0,6} I r_{0,4} / C ; P t_{0,5} I r_{0,5} / C$ em meio ácido $\left(\mathrm{H}_{2} \mathrm{SO}_{4} 0,5 \mathrm{~mol} \mathrm{~L}^{-1}\right)$ a v $=10 \mathrm{mV} \mathrm{s}^{-1}$.

de -CO são adsorvidos na superfície da platina enquanto em altos potenciais ocorre a quimissorção de grupos $-\mathrm{OH}$ durante a oxidação do álcool. Quando um segundo metal é adicionado à superfície da platina a quimissorção dos grupos - $\mathrm{OH}$ ocorre em potenciais mais baixos e se sobrepõe com a região da adsorção dos grupos $-\mathrm{CO}$ facilitando assim a oxidação do álcool.

\section{Oxidação eletroquímica de etanol}

Na Figura 5 são apresentados os voltamogramas cíclicos da eletrooxidação de etanol em meio ácido $\left(0,5 \mathrm{~mol} \mathrm{~L}^{-1} \mathrm{de}_{2} \mathrm{SO}_{4}\right)$. Todos os valores obtidos foram normalizados pela área obtida por stripping de CO. Os valores de potencial de inicio de oxidação de etanol foram medidos em 0,01 V. Os nanofios $\mathrm{Pt}_{0,9} \mathrm{Ir}_{0,1} / \mathrm{C}, \mathrm{Pt}_{0,8} \mathrm{Ir}_{0,2} / \mathrm{C}$ e $\mathrm{Pt}_{07} \mathrm{Ir}_{0,3} / \mathrm{C}$ e a Pt/C Alfa Aesar apresentaram um início de oxidação de etanol em torno de $0,53 \mathrm{~V}$. Nos nanofios $\mathrm{Pt}_{0,6} \mathrm{Ir}_{0,4} / \mathrm{C}$ e $\mathrm{Pt}_{0,5} \mathrm{Ir}_{0,5} / \mathrm{C}$ o potencial de início de oxidação ocorreu em 0,57 e 0,60 V, respectivamente. $\mathrm{O}$ resultado apresentado pelo nanofio $\mathrm{Pt}_{0,5} \mathrm{Ir}_{0,5} / \mathrm{C}$ corrobora com o valor mais positivo de potencial de início da oxidacão de $\mathrm{CO}$ observado nas análises de stripping. 


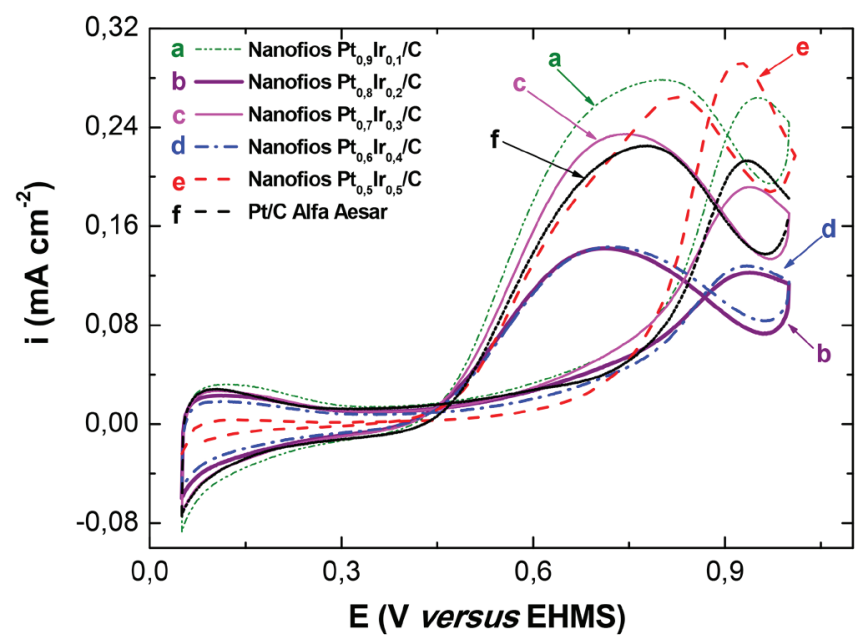

Figura 5. Voltamogramas cíclicos da oxidação eletroquímica de etanol sobre os eletrodos: Pt/C Alfa Aesar; nanofios $P t_{0,9} I r_{0,1} / C ; \mathrm{Pt}_{0,8} \mathrm{I} \mathrm{r}_{0,2} / \mathrm{C} ; \mathrm{Pt_{0,7 }} I \mathrm{I}_{0,3} / \mathrm{C}$; $\mathrm{Pt}_{0,5} \mathrm{Ir}_{0,4} / \mathrm{C} ; \mathrm{Pt}_{0,5} \mathrm{Ir}_{0,5} / \mathrm{C}$ em meio ácido $\left(\mathrm{H}_{2} \mathrm{SO}_{4} 0,5 \mathrm{~mol} \mathrm{~L}^{-1}\right)$ a $v=20 \mathrm{mV} \mathrm{s}^{-1}$.

Ramos et al., ${ }^{36}$ observaram que catalisadores ternários contendo Pt-Sn-Ir sintetizados pelo método de Pechini aumentam a oxidação de metanol e etanol, quando comparado com os catalisadores bimetálicos de Pt-Sn e Pt-Ru. As análises realizadas mostraram que o catalisador ternário continha óxidos de Sn e Ir e isso segundo os autores acaba fornecendo átomos de oxigênio que promovem a oxidação dos intermediários dos álcoois, pois esse processo age pelo mecanismo bifuncional onde os óxidos atuam como fontes de oxigênio contendo espécies para a remoção do $\mathrm{CO}$ oxidativo.

Os nanofios $\mathrm{Pt}_{0,8} \mathrm{Ir}_{0,2} / \mathrm{C}$ e apresentaram o menor valor de densidade de corrente de pico de oxidação de etanol $\left(0,12 \mathrm{~mA} \mathrm{~cm}{ }^{-2}\right)$ quando comparados com aquele observado na Pt/C Alfa Aesar $\left(0,21 \mathrm{~mA} \mathrm{~cm}^{-2}\right)$ e nos nanofios binários $\left(0,27 ; 0,19 ; 0,13\right.$ e $0,29 \mathrm{~mA} \mathrm{~cm} \mathrm{~cm}^{-2}$ para os catalisadores $\mathrm{Pt}_{0,9} \mathrm{Ir}_{0,1} / \mathrm{C}, \mathrm{Pt}_{0,7} \mathrm{Ir}_{0,3} / \mathrm{C}, \mathrm{Pt}_{0,6} \mathrm{Ir}_{0,4} / \mathrm{C}$ e $\mathrm{Pt}_{0,5} \mathrm{Ir}_{0,5} / \mathrm{C}$, respectivamente).

Note que em valores de potencial na faixa de $0,5-0,7 \mathrm{~V}$, os nanoficos com menor proporção de Ir (10, 20 e 30\%) apresentam valores de densidade de corrente maiores aos mostrados pelos demais catalisadores estudados.

Na Figura 6 estão representadas as curvas cronoamperométricas dos nanofios sinstetizados. Uma diminuição das correntes em função do tempo é observada para todos os eletrocatalisadores. Imediatamente após a aplicação do potencial inicial, a corrente decresce rapidamente devido ao carregamento da dupla camada e a ocorrência de outros processos sobre a superfície do eletrodo. Este decréscimo é seguido de mudanças em direção a um estado quase estacionário depois de aproximadamente 250 segundos para Pt Alfa Aesar, 500 e 750 segundos para nanofios $\mathrm{Pt}_{0,9} \mathrm{Ir}_{0,1} / \mathrm{C}$ e $\mathrm{Pt}_{0,8} \mathrm{Ir}_{0,2} / \mathrm{C}$ respectivamente. Quanto mais rápido o catalisador atingir o estado estacionário e quanto menor for a perda de corrente, mais estável será o catalisador. Pode-se observar que esse decaimento brusco seguido de um decaimento lento nos primeiros $500 \mathrm{~s}$, pode ser devido à eliminação de impurezas superficiais resultantes da oxidação incompleta do álcool e alcançando uma estabilidade nos minutos finais. ${ }^{37}$

Os valores obtidos das pseudo-densidades de corrente de equilíbrio ao final de $3600 \mathrm{~s}$ para os eletrocatalisadores de $\mathrm{Pt}_{0,9} \mathrm{Ir}_{0,1} / \mathrm{C}$; $\mathrm{Pt}_{0,8} \mathrm{Ir}_{0,2} / \mathrm{C} ; \mathrm{Pt}_{0,7} \mathrm{Ir}_{0,3} / \mathrm{C} ; \mathrm{Pt}_{0,6} \mathrm{Ir}_{0,4} / \mathrm{C} ; \mathrm{Pt}_{0,5} \mathrm{Ir}_{0,5} / \mathrm{C}$ e Pt/C Alfa Aesar durante a oxidação de etanol foram: 3,$83 ; 3,62 ; 3,10 ; 2,47 ; 1,43$ e $-0,13 \mathrm{~mA}$ $\mathrm{cm}^{-2}$, respectivamente. Constatando-se que os eletrocatalisadores sintetizados apresentaram um rendimento superior à Pt/C comercial (Alfa Aesar). Resultados semelhantes foram obtidos por Ayoub et al. ${ }^{37} \mathrm{e}$ Spinacé et al..$^{38}$



Figura 6. Curvas cronoamperométricas de etanol sobre os eletrodos: $P t / C \mathrm{da}$ Alfa Aesar; nanofios $P t_{0,9} I r_{0,1} / C ; P t_{0,8} I r_{0,2} / C ; P t_{0,7} I r_{0,3} / C ; P t_{0,6} I r_{0,4} / C ; P t_{0,5} I r_{0,5} / C$ em meio ácido $\left(\mathrm{H}_{2} \mathrm{SO}_{4} 0,5 \mathrm{~mol} \mathrm{~L} \mathrm{~L}^{-1}\right)$ em potencial anódico de $0,5 \mathrm{~V}$ durante $3600 \mathrm{~s}$.

A capacidade anti-envenenamento dos eletrocatalisadores é um importante parâmetro a ser abordado antes da utilização em aplicações comerciais. Os eletrocatalisadores sintetizados neste estudo se mostraram eficientes frente à oxidação de etanol quando comparados com a Pt/C da Alfa Aesar. Antes da reação catalítica, os locais eletroquimicamente ativos são acessíveis para a adsorção das moléculas de etanol. Posteriormente, a adsorção de novas moléculas dependeria dos locais ativos liberados ocupados pelas espécies intermediárias do tipo CO geradas no processo de oxidação de etanol, afetando eventualmente a cinética da eletrocatálise.

\section{CONCLUSÕES}

Pode-se concluir que a rota de síntese empregada para os eletrocatalisadores do tipo nanofios $\mathrm{Pt}-\mathrm{Ir} / \mathrm{C}$ cristalinos utilizando o método de redução dos íons precursores metálicos com ácido fórmico como agente redutor foi eficaz conforme os resultados de difração de raios $X$.

Os resultados de FRX para os nanofios sintetizados por este método mostraram que os valores das proporções atômicas são similares as nominais. As variações nas proporções atômicas dos metais Pt e Ir (90:10), (80:20), (70:30), (60:40) e (50:50) foram fatores determinantes para o aumento da atividade catalítica dos nanofios sintetizados neste trabalho, quando comparados com a Pt comercial da Alfa Aesar em todos os casos. Os padrões de difração de raios X mostraram os planos cristalinos característicos da estrutura cúbica de faces centrada da platina e suas ligas, além disso, pode-se observar que há um indicativo da formação da liga Pt-Ir/C já que não foram observadas mudanças significantes nos padrões de DRX com a incorporação do irídio.

As análises de MET mostraram que através da rota de síntese empregada foi possível obter nanofios com diâmetros variando entre 4 e $7 \mathrm{~nm}$ e um comprimento dos fios de 10 a $20 \mathrm{~nm}$. Com a formação dos nanofios interconectados, mais defeitos são gerados com locais de reação extra-ativos apresentando uma melhor tolerância ao $\mathrm{CO}_{\mathrm{ads}}$, pois o mesmo pode ser oxidado com maior facilidade.

As análises de stripping de $\mathrm{CO}$ apresentaram que a maioria dos nanofios de $\mathrm{Pt}-\mathrm{Ir} / \mathrm{C}$ oxidaram o $\mathrm{CO}$ em potenciais mais negativos em comparação a Pt/C da Alfa Aesar e isso pode ser explicado devido ao $\mathrm{CO}$ ser oxidado em locais adjacentes. Assim, essa alta mobilidade dos adsorventes $\left(\mathrm{CO}_{\text {ads }}\right.$ e $\left.\mathrm{OH}_{\text {ads }}\right)$ facilitaria a reação já que os nanofios 
apresentam uma ligação mais fraca com as espécies contendo oxigênio em relação aos nanopartículas.

Nanofios com baixas proporções de Ir (entre 10 e 30\%) se mostraram bastante eficientes na eletrooxidação de etanol, indicando que esses catalisadores possuem maior número de sítios ativos disponíveis para a adsorção das moléculas de etanol a baixos potenciais. Assim, os nanofios sintetizados neste trabalho foram ativos em baixos potenciais para eletrooxidação de etanol, ou seja, potenciais dentro da faixa aceitável para aplicação tecnológica, podendo ser recomendados para aplicação prática em células a combustível de etanol direto.

\section{AGRADECIMENTOS}

Ao CNPq (processos 305438/2018-2 e 310282/2013-6), CAPES (código de financiamento 001) e Fapitec/SE.

\section{REFERÊNCIAS}

1. Moghaddam, E. A.; Ahlgren, S.; Hulteberg, C.; Nordberg, A.; Fuel Process. Technol. 2015, 132, 74.

2. Disano, J.; Indicators of sustainable development: Guidelines and methodologies, 2001.

3. Hossain, Md.; F.; Int. J. Environ. Sci. Te. 2019, 16, 755.

4. Liyuan, G.; Zhiyuan, Y.; Li, K.; Xing, W.; Liu, C., Ge, J.; J. Energy Chem. 2018, 27, 1618

5. Ong, B. C.; Kamarudin, S. K.; Basri, S.; Int. J. Hydrogen Energ., 2017, 42, 10142

6. Saadabadi, S. A.; Thattai, A. T.; Fan, L.; Lindeboom, R. E. F.; Spanjers, H.; Aravind, P. V.; Renew. Energ., 2019, 134, 194.

7. Pana, Z. F.; Ana, L.; Zhaob, T. S.; Tang, Z. K.; Prog. Energ. Combust., 2018, 66, 141175 .

8. Abdullah, N.; Kamarudin, S. K.; J. Power Sources, 2015, 278, 109.

9. Nižeti, S.; Tolj, I.; Papadopoulos, A. M.; Energy Convers Manage, 2015, 05, 1037.

10. Brouzgou, A.; Podias, A.; Tsiakaras, P.; J. Appl. Electrochem., 2013, 43, 119.

11. Shlapbach, L.; Zuettel, A.; Nature, 2001, 414, 353.

12. Limayem, A.; Rick, S. C.; Prog. Energ. Combust., 2012, 38, 449.

13. Prieto, M. J.; Tremiliose-Filho, G.; Electrochem. Commun., 2011, 13 , 527.

14. Zhao, L.; Mitsushima, S.; Ishihara, A.; Matsuzawa, K.; Ota, K.; Chinese Journal of Catalysis, 2011, 32, 1856.

15. Cao, L.; Sun, G.; Li, H.; Xin, Q.; Electrochem. Commun., 2007, 9, 2541.

16. Tayal, J.; Rawat, B.; Basu, S.; Int. J. Hydrogen Energ. 2011, 36, 14884.

17. Zhou, W.P.; Li, M.; Koenigsmann, C.; Ma, C.; Wong, S. S.; Adzic, R. R.; Electrochimica Acta, 2011, 56, 9824.
18. Kamarudin, S. K.; Hashim N.; Renew. Sust. Energ. Rev. 2012, 16, 2494.

19. Almeida, G. R. O.; Sussuchi, E. M.; Meneses, C. T.; Salazar-Banda, G. R.; Eguiluz, K. I. B.; Int. J. Electrochem. Sci., 2017, 12, 7502.

20. Neto, E. S. V.; Gomes, M. A.; Salazar-Banda, G. R.; Eguiluz, K. I. B.; Int. J. Hydrogen Energ. 2018, 43, 178.

21. Almeida, G. R. O.; López-Suárez, F. E.; Silva, L. S. R.; Pereira, G. F.; Bueno-López, A.; Salazar-Banda, G. R.; Eguiluz, K. I. B.; J. Nanosci. Nanotechnol., 2019, 19, 795.

22. Zhang, T.; Li, S.-Ch.; Zhu, W.; Zhang, Z.-P.; Gu, J.; Zhang, Y.-W.; Nanoscale, 2017, 9, 1154

23. Young, R. A.; Larson, A. C.; Paiva-Santos, C. O. Program DBWS9807A - Rietveld analysis of X-ray and neutrons powder diffraction patterns, User's Guide, 2000.

24. Calderón, J. C.; García G.; Calvillo, L.; Rodríguez, J. L.; Lázaro, M. J.; Pastor E., Appl Catal B Environ. 2015, 165, 676.

25. Montero, M. A.; Fernández, J. L.; de Chialvo, M. R. G.; Chialvo, A. C.; J Phys Chem C. 2013, 117, 25269.

26. Wendt, H.; Spinacé, E. V.; Neto, A. O.; Linardi, M.; Quim. Nova, 2005, $28,1066$.

27. Tahmasebi, S.; McMath, A. A.; van Drunen, J.; Jerkiewicz, G.; Electrocatalysis, 2017, 8, 301.

28. Korotkikh, O.; Farrauto, R.; Catal. Today, 2000, 62, 249.

29. Ciapina, E. G.; Santos, S. F.; Gonzalez, E. R.; J. Electroanal. Chem. 2018, 815, 47 .

30. Ochal, P.; La Fuente, J. L. G. D.; Tsypkin, M.; Seland, F.; Sunde, S.; Muthuswamy, N.; Rønning, M.; Chen, De.; Garcia, Sergio.; Alayoglu, S.; Eichhorn, B.; J. Electroanal. Chem. 2011, 655, 140

31. Batista, E. A.; Iwasita, T.; Vielstich W.; J. Phys. Chem. B, 2004, 108, 14216

32. Qu, Y.; Wang, L.; Li, C.; Gao, Y.; Kyong Sik, J.; Rao, J.; Yin, G.; Int. J. Hydrogen Energ., 2017, 42, 228.

33. Rau, M. S.; Chialvo, M. R. G.; Chialvo, A. C.; J. Solid State Electr., 2012, 16, 1893 .

34. Yuan, J.; He, B.; Hong, L.; Lu, J.; Miao, J.; Niu, L.; J. Mater. Chem., 2012, 22, 19658.

35. Shivhare, M. R.; Allen, R. G.; Scott, K.; Morris, A. J.; Martin, E. B.; J. Electroanal. Chem., 2006, 595, 145.

36. Ramos, S. G.; Calafiore, A.; Bonesi, A. R.; Triaca, W. E.; Luna, A. M. C.; Moreno, M. S.; Zampieri, G.; Bengio, S.; Int. J. Hydrogen Energ., 2012, 37, 14849.

37. Ayoub, J. M. S.; Geraldes, A. N.; Tusi, M. M.; Spinacé, E. V.; Neto, A. O.; Ionics, 2011, 17, 559.

38. Spinacé, E. V.; Dias, R. R.; Brandalise, M.; Linardi, M.; Neto, A. O.; Ionics, 2010, 16, 91 . 